\title{
Simulation of Effective Slip and Drag in Pressure-Driven Flow on Superhydrophobic Surfaces
}

\author{
Yuanding Huang, Xuezeng Zhao, Yunlu Pan, and Khurshid Ahmad \\ Key laboratory of Micro-Systems and Micro-Structures Manufacturing, Ministry of Education, \\ School of Mechatronics Engineering Harbin Institute of Technology, Harbin 150001, China \\ Correspondence should be addressed to Yunlu Pan; yunlupan@hit.edu.cn
}

Received 18 September 2015; Revised 6 January 2016; Accepted 10 January 2016

Academic Editor: Waqar A. Khan

Copyright (C) 2016 Yuanding Huang et al. This is an open access article distributed under the Creative Commons Attribution License, which permits unrestricted use, distribution, and reproduction in any medium, provided the original work is properly cited.

\begin{abstract}
The flow on superhydrophobic surfaces was investigated using finite element modeling (FEM). Surfaces with different textures like grooves, square pillars, and cylinders immersed in liquid forming Cassie state were modeled. Nonslip boundary condition was assumed at solid-liquid interface while slip boundary condition was supposed at gas-liquid interface. It was found that the flow rate can be affected by the shape of the texture, the fraction of the gas-liquid area, the height of the channel, and the driving pressure gradient. By extracting the effective boundary slip from the flow rate based on a model, it was found that the shape of the textures and the fraction of the gas-liquid area affect the effective slip significantly while the height of the channel and the driving pressure gradient have no obvious effect on effective slip.
\end{abstract}

\section{Introduction}

Development in microfluidics has stimulated interest in manipulating flows in micro/nanoscale channels $[1,2]$. Drag reduction at the solid-liquid interface is an important challenge in the research and application of microfluidics. Boundary slip can reduce the drag at the interface [3-7]. Boundary slip is quantified by slip length $b$, which is the distance beyond the solid-liquid interface at which the velocity profile extrapolates to zero [7-9]. Researches have shown that flat, smooth, and chemically homogenous hydrophilic surfaces exhibit no or less slip [10, 11]. However, if the surface is hydrophobic, there can be slip with the magnitude up to tens of nanometers $[6,12-15]$. In the meanwhile, superhydrophobic surfaces, on which the contact angle exceeds $150^{\circ}$ and the contact angle hysteresis is less than $5^{\circ}$, have been proved to be able to significantly amplify the magnitude of slip. These surfaces have composite surface structures, which can trap gas at the grooves or pits $[16,17]$ and form a Cassie state. The slip length on superhydrophobic surface or other complex heterogeneous surfaces is represented by effective slip $\left(b_{\text {eff }}\right)$. The $b_{\text {eff }}$ is obtained by averaging the flow over the characteristic length scale of the configuration (e.g., a channel) [4], which is usually defined as

$$
b_{\text {eff }}=\frac{\left\langle u_{s}\right\rangle}{\left\langle(\partial u / \partial z)_{s}\right\rangle},
$$

where $\langle\cdots\rangle$ means the average value in the supposed plane $x O y$ on surfaces [18]. Experiments of flow on superhydrophobic surfaces have indicated that the $b_{\text {eff }}$ can reach to the order of several or tens of microns [19-24].

As most of the "super" properties of superhydrophobic surfaces are due to the gas entrapment, the Cassie state is preferred rather than the Wenzel state. Numerous research efforts have been done during the last few decades in order to stabilize the Cassie state $[21,25,26]$. Since that it is hard to measure the actual fraction of solid in contact with liquid for arbitrary rough or fractional surface, surfaces with well controlled periodic textures have been widely used [27].

In recent years, the researches focused on quantitative understanding of the effective slippage on the superhydrophobic surfaces. Attention has been paid to surfaces with directional patterns, such as the arrays of parallel 
TABLE 1: Parameters of pressure-driven flow model (unit: $\mu \mathrm{m}$ ).

\begin{tabular}{lccr}
\hline Fixed parameter & Value & Variable Parameter & \\
\hline Length and width of the channel $L \times W$ & $60 \times 60$ & Height of the channel $H$ & Value \\
Period length of the textures $D$ & 10 & Size of the textures $d$ & Range $(1,1,100)$ \\
Height of the textures $h$ & $\sim$ & Interval $w$ & Range $(0.2,0.2,9.8)$ \\
\hline
\end{tabular}

${ }^{*} x, y$, and $z$ in "range $(x, y, z)$ " represent the initial value, step length, and final value.

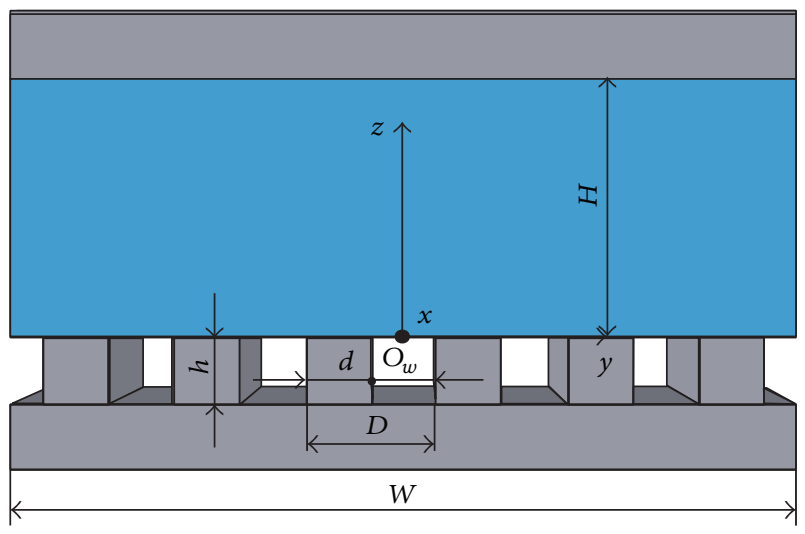

FIgURE 1: Schematic of pressure-driven flow model.

superhydrophobic grooves, square pillars, and cylinders that generate anisotropic effective slip in the Cassie regime experimentally and theoretically [17, 28-31]. This paper extended the work to investigate the effective slip as well as drag in pressure-driven flow on superhydrophobic surfaces decorated with arrays of grooves, square pillars, and cylinders, respectively, through simulation. The flow has been studied with the help of FEM technique and the results as well as discussion are given in the following sections.

\section{Models and Calculation}

2.1. Models. Surfaces in Cassie state with textures in micro scale usually lead to the so-called superhydrophobicity. Three different textures, that is, grooves, square pillars, and cylinders, were chosen to decorate the surface.

In most of the microfluidic applications, the flow is driven through pressure. Therefore, pressure-driven fluid that flows through a straight channel was modeled, in which the upper plate is an ideal flat one, while the lower one is the superhydrophobic surfaces with special textures (Figure 1). Fluid flows through the channel, fully wetting the upper plate but partially wetting the lower plate, which form the Cassie state. To simplify the model, the meniscus curvature at the gas-liquid interface was ignored, as considered in most previous publications $[6,13,32-34]$. The length $L$ and width $W$ of the channel, the period length $D$, and the height $h$ of the textures are in constant number as shown in Table 1 . The size of the textures $d$, the width of the interval $w(w+d=D)$, and the height of the channel $H$ are main variables. Using these parameters, the fraction of gas-liquid area $\Phi_{g}$ can be calculated.
Figure 2 shows that the fluid flows from one side to the opposite side. According to the flow direction, there can be five typical flow regimes over these textures: (a) longitudinal direction of grooves, (b) transverse direction of the grooves, (c) parallel (or perpendicular) to the array of the square pillars, (d) $45^{\circ}$ to the array of the square pillars, and (e) parallel (or perpendicular) to the array of the cylinders.

2.2. Fluid Properties. Incompressible water was chosen as the fluid, of which the flow follows the Navier-Stokes equations and the mass continuity equation:

$$
\begin{aligned}
\rho(\mathbf{u} \cdot \nabla) \mathbf{u} & =\nabla \cdot\left[-p \mathbf{I}+\mu\left(\nabla \mathbf{u}+(\nabla \mathbf{u})^{T}\right)\right]+\mathbf{F}, \\
\rho \nabla \cdot(\mathbf{u}) & =0
\end{aligned}
$$

where $\rho$ is the density of the fluid, $\mathbf{u}$ is the of velocity vector, $p$ is the driven pressure, $\mathbf{I}$ is the momentum vector, $\mu$ is the dynamic viscosity of the fluid, and $\mathbf{F}$ is the external force. The flow field remains laminar due to microscale and the low driven pressure. The external reference pressure is 1 bar $\left(1.01325 \times 10^{5} \mathrm{~Pa}\right)$, and room temperature is $293.15 \mathrm{~K}$, the density of water $\rho=0.9982071 \times 103 \mathrm{~g} / \mathrm{cm}^{3}$, and dynamic viscosity $\mu=1.0050 \times 10^{-3} \mathrm{~Pa} \cdot \mathrm{s}$ [32].

2.3. Boundary Condition. In the so-called "gas cushion model," the interfacial region is described as a lubricating "gas film" of thickness $e$ and viscosity $\mu_{g}$, while the bulk value is $\mu$. The slip can be calculated [33]

$$
b=e\left(\frac{\mu}{\mu_{\mathrm{g}}}-1\right) \approx e \frac{\mu}{\mu_{\mathrm{g}}} .
$$

When the thickness of gas film ranges between 0.1 and $10 \mu \mathrm{m}$, the slip at the gas-liquid interface is about $5-500 \mu \mathrm{m}$ (dynamics viscosity of gas $\mu_{g}=1.8107 \times 10^{-5} \mathrm{~Pa} \cdot \mathrm{s}$ at $293.15 \mathrm{~K}$ [33]). This model is justified for a continuous "gas film" on a homogeneous surface [32] or surface with shallow textures [34]. Note that it has been verified that the slip length at the smooth solid-liquid interface is no more than tens of nanometers. Therefore, the slip at the upper flat plate (Figure 1) can be ignored. Accordingly, this simulation has set the boundary conditions to nonslip at the upper plate as well as at the top of the textures, as described by (4). It has been assumed that the slip only occurs at the space between the textures, expressed by (5). The slip condition depends on the contact state at the superhydrophobic surface. It is confined 


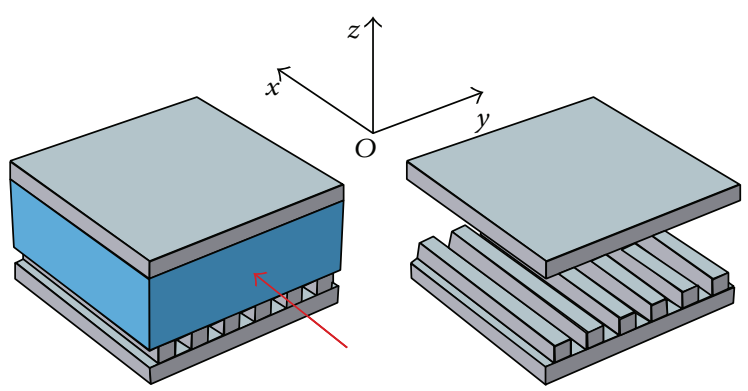

(a)

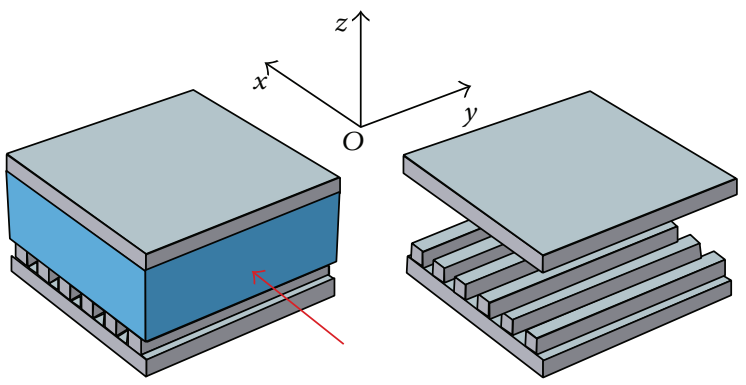

(b)

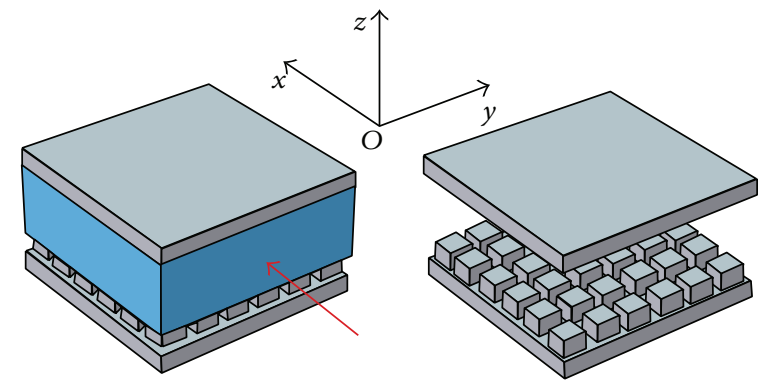

(c)

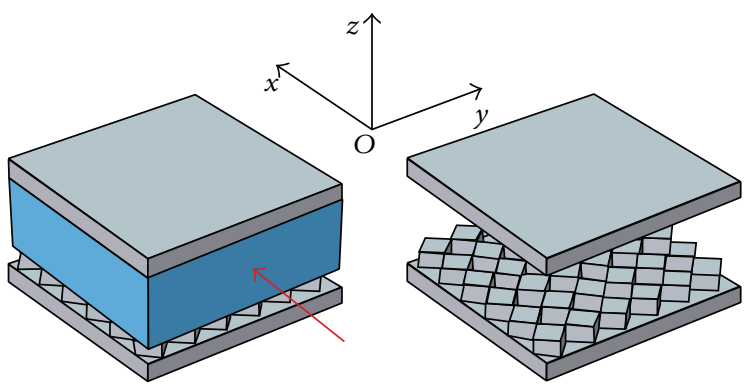

(d)

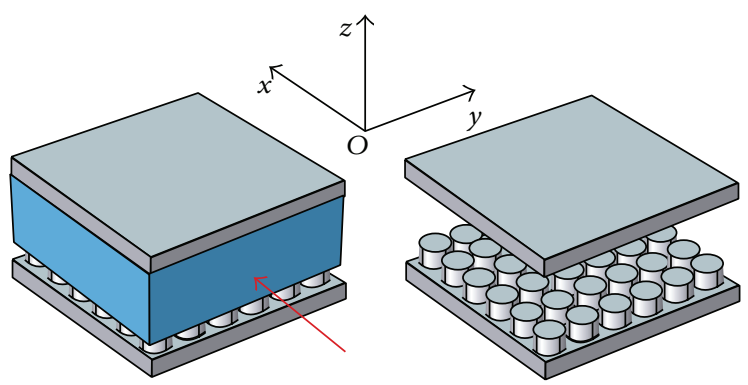

(e)

FIGURE 2: States of pressure-driven flow models: longitudinal grooves (a), transverse grooves (b), square pillars $0^{\circ}$ (c), square pillars $45^{\circ}$ (d), and cylinders (e). 

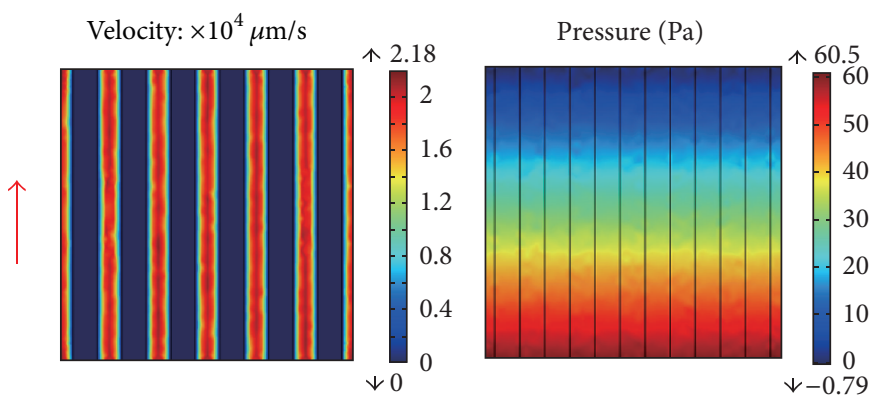

(a)

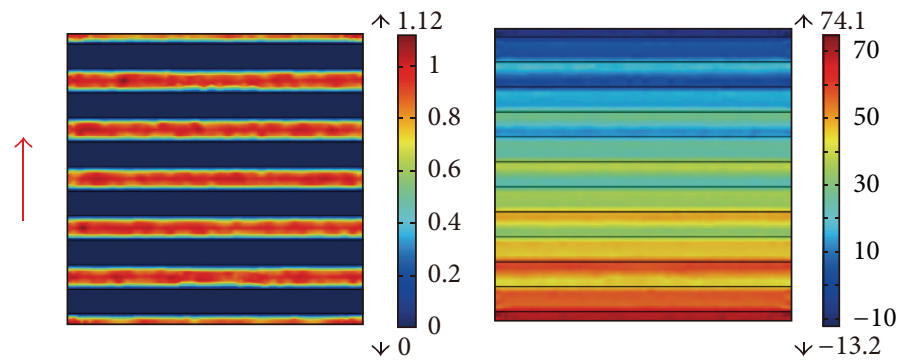

(b)
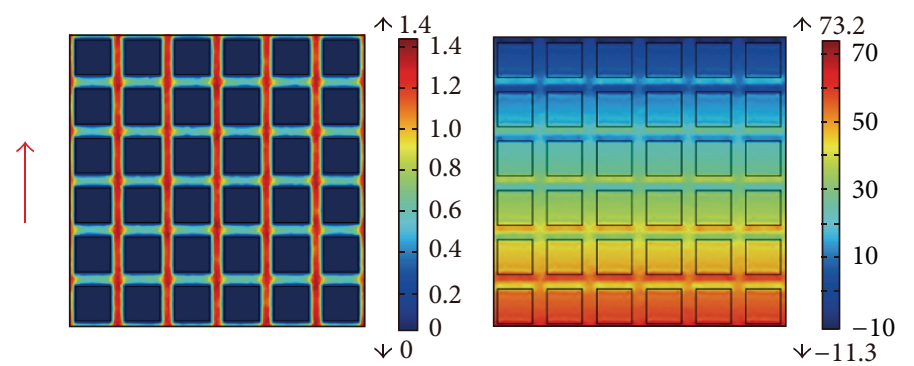

(c)
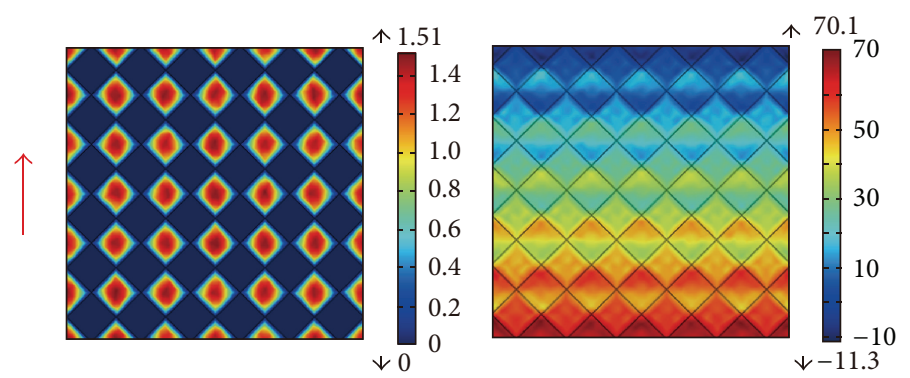

(d)

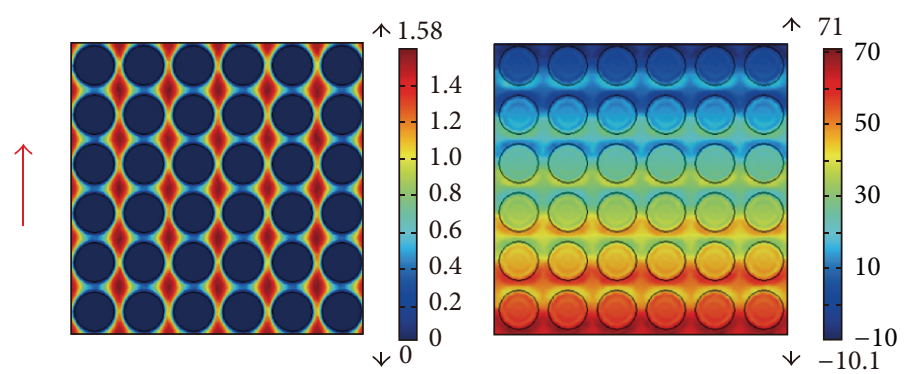

(e)

FIGURE 3: Distributions of velocity and pressure at the interface of superhydrophobic surface and liquid: longitudinal grooves (a), transverse grooves (b), square pillars $0^{\circ}(\mathrm{c})$, square pillars $45^{\circ}(\mathrm{d})$, and cylinders (e). 
by the geometry of gas-liquid interface rather than the depth of the textures. Equations (4) and (5) take the form

$$
\begin{aligned}
\mathbf{u}=0, \\
\mathbf{u} \cdot \mathbf{n}=0, \\
\mathbf{K}-(\mathbf{K} \cdot \mathbf{n}) \mathbf{n}=0,
\end{aligned}
$$

where $\mathbf{n}$ is the normal vector and $\mathbf{K}=\left[\mu\left(\nabla \mathbf{u}+(\nabla \mathbf{u})^{T}\right)\right] \mathbf{n}$. These boundary conditions assume that the gas layer has no friction force to the liquid flow but confine the fluid velocity direction parallel to the wall.

Fluids at the inlet and the outlet are all of normal flow without backflow, following

$$
\begin{aligned}
\mathbf{n}^{T}\left[-p \mathbf{I}+\mu\left(\nabla \mathbf{u}+(\nabla \mathbf{u})^{T}\right)-\frac{2}{3} \mu(\nabla \cdot \mathbf{u}) \mathbf{I}\right] \mathbf{n} & =-\widehat{p}_{0}, \\
\mathbf{u} \cdot \mathbf{t} & =0
\end{aligned}
$$

where $\widehat{p}_{0}$ is the pressure at the outside of the fluid field near inlet or outlet, $\widehat{p}_{0} \geq p_{\text {in }}$ at the inlet, and $\widehat{p}_{0} \leq p_{\text {out }}$ at the outlet; $\mathbf{t}$ is the tangential vector to the wall. In many previous publications about pressure-driven microchannel flow $[15,35,36]$, the pressure gradient is assumed $d p / d x=$ $-1 \times 10^{-6} \mathrm{~N} / \mathrm{m}^{3}$. With reference to this, in this model, $p_{\text {in }}$ is set a variable parameter ranging from 6 to $600 \mathrm{~Pa}$ with the step length of $6 \mathrm{~Pa}$, while $p_{\text {out }}=0$; in which case, $d p / d x$ ranges from $-1 \times 10^{5}$ to $-1 \times 10^{7} \mathrm{~N} / \mathrm{m}^{3}$ with the step length $-1 \times 10^{5} \mathrm{~N} / \mathrm{m}^{3}$. Moreover, the lateral sides of the fluid flow field are defined by symmetric plane.

The flow was simulated in Comsol Multiphysics. The effect of the shape of textures, fraction of gas, height of channel, and driving pressure gradient on effective slip as well as drag was studied. Five different cases, as shown in Figure 1, were simulated. The results are given in the corresponding sections. Moreover, it has set that the flow driven by pressure in microchannel with superhydrophobic surface still follows Navier-Stokes equation, but with an effective slip $b_{\text {eff }}$ on the superhydrophobic surface, described as

$$
\begin{aligned}
\mu \frac{\partial^{2} u}{\partial z^{2}} & =\frac{d p}{d x}, \\
\left.u\right|_{z=H / 2} & =0, \\
\frac{\left.u\right|_{z=-H / 2}}{b_{\text {eff }}} & =\left.\frac{\partial u}{\partial z}\right|_{z=-H / 2} .
\end{aligned}
$$

The flow rate in the channel is calculated by

$$
Q=\int_{H / 2}^{-H / 2} u d z \cdot W
$$

Combining (7) and (8), it can get relationship between effective slip $b_{\text {eff }}$ and flow rate $Q$ :

$$
b_{\text {eff }}=-\frac{12 \mu H Q+(d p / d x) H^{4} W}{4(d p / d x) H^{3} W+12 \mu Q} .
$$

Therefore, the effective slip $b_{\text {eff }}$ can be obtained through above equation as the flow rate $Q$ is obtained through the simulation.

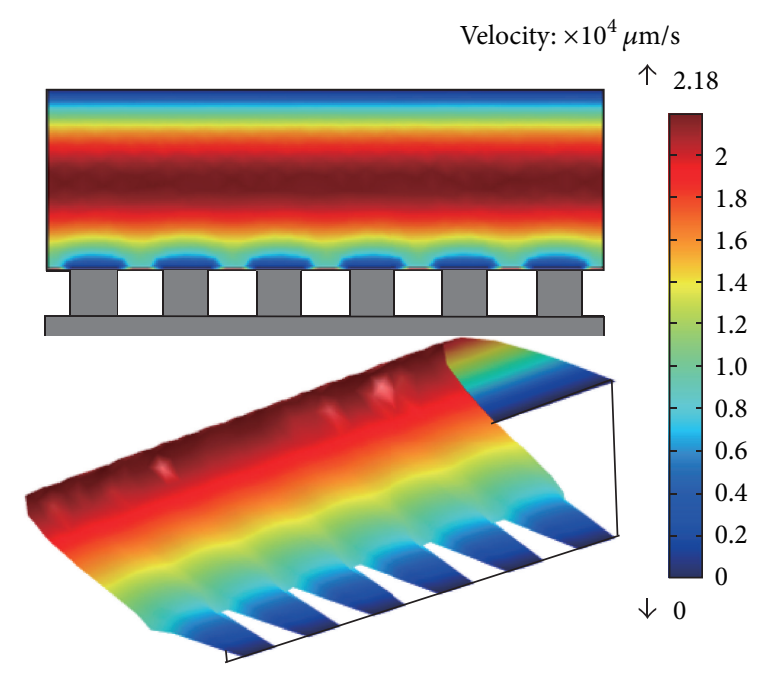

FIGURE 4: Distribution of velocity at the longitudinal cross section perpendicular to the direction of flow.

\section{Results and Discussions}

3.1. Effect of the Surface Textures. The interface at flat surface or the top of the textures refers to a solid-liquid contact while the interface of the gap and the liquid refers to a gas-liquid contact. A nonslip condition was supposed at the solid-liquid interface while a slip condition was assumed at gas-liquid interface. As an example, the distributions of velocity and pressure of the fluid at the interface of superhydrophobic surfaces with $\Phi_{g}=0.5, H=20 \mu \mathrm{m}$, and $d p / d x=-1 \times 10^{-6} \mathrm{~N} /$ $\mathrm{m}^{3}$ are shown in Figure 3 . The velocity at the gas-liquid interface is higher than that at the solid-liquid interface; however, the shape of the textures still affects the distribution and magnitude of velocity of the fluid. If the textures have small angle of inclination to the direction of flow, there will be lower drag; thus the flow can be enhanced. It can be noticed that the liquid flows smoothly on the longitudinally grooved surface. However, the flow on surfaces textured with square pillars or cylinders changes with the angle of inclination. The drag will be larger with larger angle of inclination.

Figure 4 shows the flow distribution in the direction perpendicular to the flow. The velocity near the walls is lower while it is higher at the center of the channel. Noticeably, at the contact line of superhydrophobic surface and liquid, the velocity is zero at the solid-liquid interface while it is large at the gas-liquid interface where it shows a finite slip.

3.2. Effect of the Gas-Liquid Fraction. Different sized textures were modeled on the surfaces shown in Table 1 . The effect of the gas-liquid fraction on the flow rate and effective slip are shown in Figures 5 and 6, respectively. $Q_{s} / Q_{f}$ is used to show the change of the flow rate where $Q_{s}$ is the flow rate in microchannels with textured surfaces and $Q_{f}$ is the flow rate in microchannels with smooth surfaces in the same condition. Due to the constraint of the shape, the fraction of gas-liquid area $\Phi_{g}$ of surfaces decorated with square pillars with the angle of inclination $45^{\circ}$ cannot be lower than 
TABLE 2: Average and standard deviation of effective slip in study of effect of height (unit: $\mathrm{nm}$ ).

\begin{tabular}{|c|c|c|c|c|c|}
\hline $\begin{array}{l}H \in(20,100) \mu \mathrm{m} \\
\Phi_{g}=0.5 ; d p / d x=-1 \times 10^{6} \mathrm{~N} / \mathrm{m}^{3}\end{array}$ & Longitudinal grooves & Transverse grooves & Square pillars $0^{\circ}$ & Square pillars $45^{\circ}$ & Cylinders \\
\hline$b_{\text {eff }}$ & $733.0 \pm 49.4$ & $277.7 \pm 29.4$ & $227.6 \pm 31.0$ & $394.3 \pm 31.4$ & $342.8 \pm 25.7$ \\
\hline
\end{tabular}

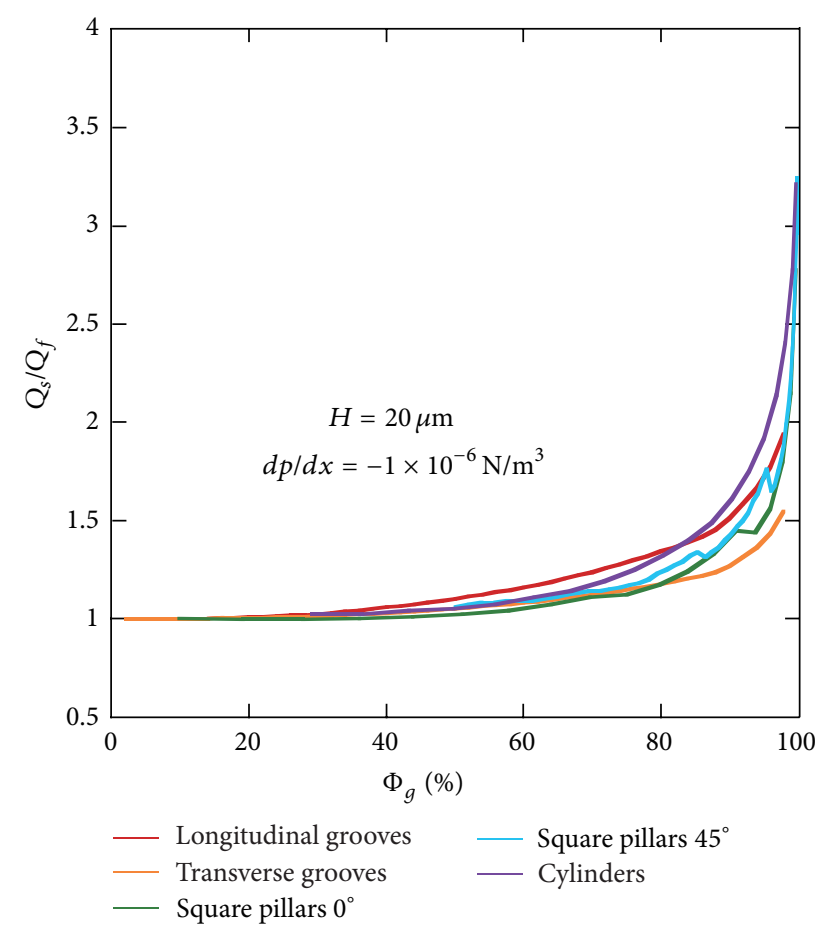

FIGURE 5: Relational graph of fraction of gas-liquid area to the flow rate.

$50 \%$, while that surface textured with cylinders should be larger than about $21 \%$. Also, surfaces decorated with certain patterns, on which the fraction of gas-liquid area can be up to 99\%, have been realized and applied in experiment [20].

As shown in Figures 5 and 6, the lines of flow rate and effective slip in the same regime show a similar trend and increase exponentially with the increase of $\Phi_{g}$. When $\Phi_{g}$ is lower than about $84 \%$, the flow rate and effective slip on the surfaces with longitudinal grooves are larger than the other surfaces. When $\Phi_{g}$ increases over $90 \%$, the enhancement of the flow in the other surfaces is more significant than the channel with transverse grooves decorated surface. The obstruction of the flow by the transverse grooves may be the main reason of the weak enhancement of flow rate. Moreover, the magnitude of the effective slip ranges in nanometers when the fraction is lower than $50 \%$, and it reaches to about 3$57 \mu \mathrm{m}$ when the fraction is larger than $90 \%$, which is in agreement with the previous experimental as well as theoretical results [19-24]. Maali et al. [19] got $291 \mathrm{~nm}$ effective slip length on cylinders decorated surface with a $\Phi_{g}=0.63$ measured by AFM, while it shows about $700 \mathrm{~nm}$ effective slip in this simulation in the same state. Choi et al. [20] studied the effect of gas fraction of textured hydrophobic surfaces on slip using rheometer system, and they obtained about $18 \mu \mathrm{m}, 25 \mu \mathrm{m}$,

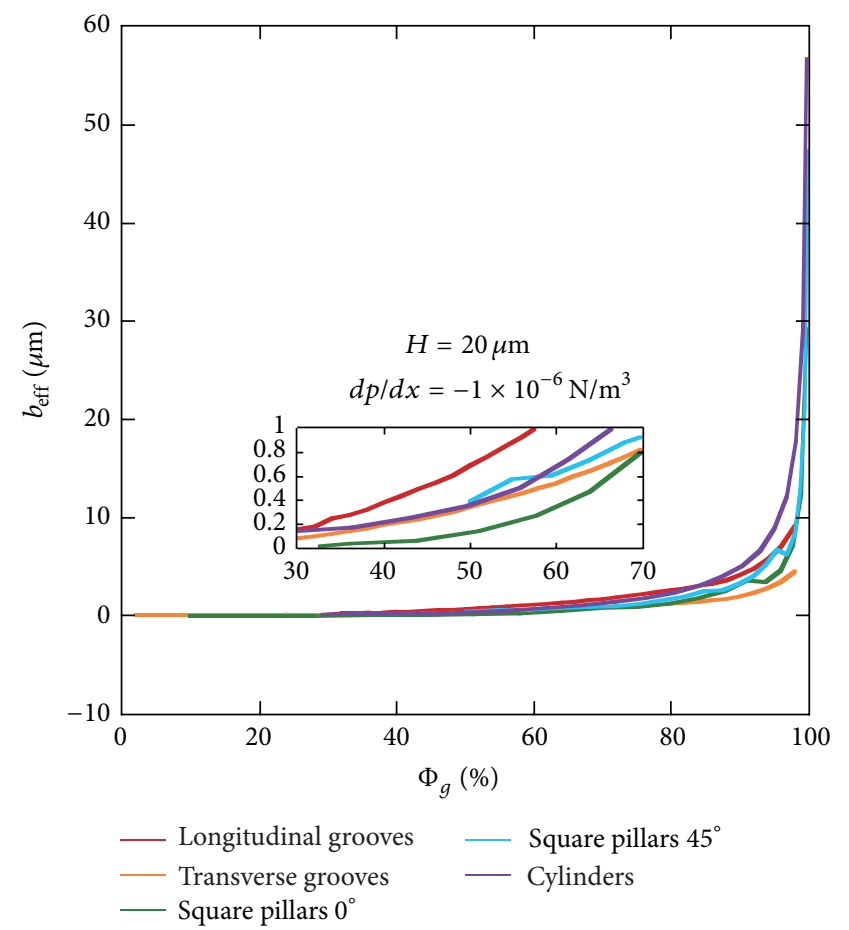

FIGURE 6: Relational graph of fraction of gas-liquid area to the effective slip.

$50 \mu \mathrm{m}, 80 \mu \mathrm{m}$, and $100 \mu \mathrm{m}$ slip length at the gas fraction of $50 \%, 85 \%, 95 \%, 98 \%$, and $99 \%$ on surface textured with cylinders, respectively. At the same fraction of gas-liquid area, this simulation shows $350 \mathrm{~nm}, 3 \mu \mathrm{m}, 8 \mu \mathrm{m}, 17 \mu \mathrm{m}$, and $29 \mu \mathrm{m}$ slip length, respectively. Although the numbers of effective slips have deviations, the trends of the boundary slip with the fraction are in agreement. The inaccuracy of experiments $[12,37]$, the existence of meniscus at the gas-liquid interface [38-40], and the simplifications in this simulation might be the reason of the deviations on the numbers.

3.3. Effect of the Height of the Channel and Driving Pressure. The effect of the height of the channel $H$ on the flow rate was also studied. The gas-liquid fraction $\Phi_{g}$ was fixed at 0.5 while the driving pressure gradient $d p / d x=-1 \times 10^{-6} \mathrm{~N} / \mathrm{m}^{3} . H$ ranged from 1 to $100 \mu \mathrm{m}$ with the step length $1 \mu \mathrm{m}$. The results are shown in Figures 7 and 8 and Table 2.

As shown in Figure 7, the flow rate in channels characterized with textures can reach twice the flow rate on smooth surface when $H$ is as small as $1 \mu \mathrm{m}$, and the enhancement decreases rapidly with the increase of $H$ and the flow rates on all textured surfaces are nearly the same as the flow rate on smooth surface when $H$ reaches $100 \mu \mathrm{m}$. It strongly suggests 


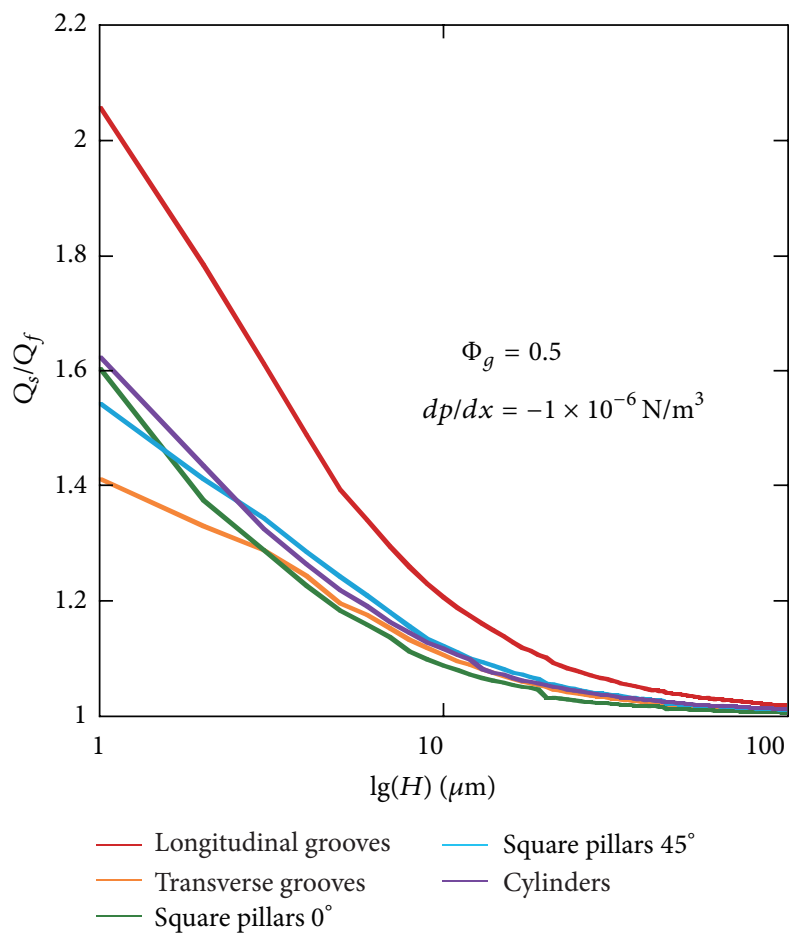

FIGURE 7: Relational graph of height of the channel to the flow rate.

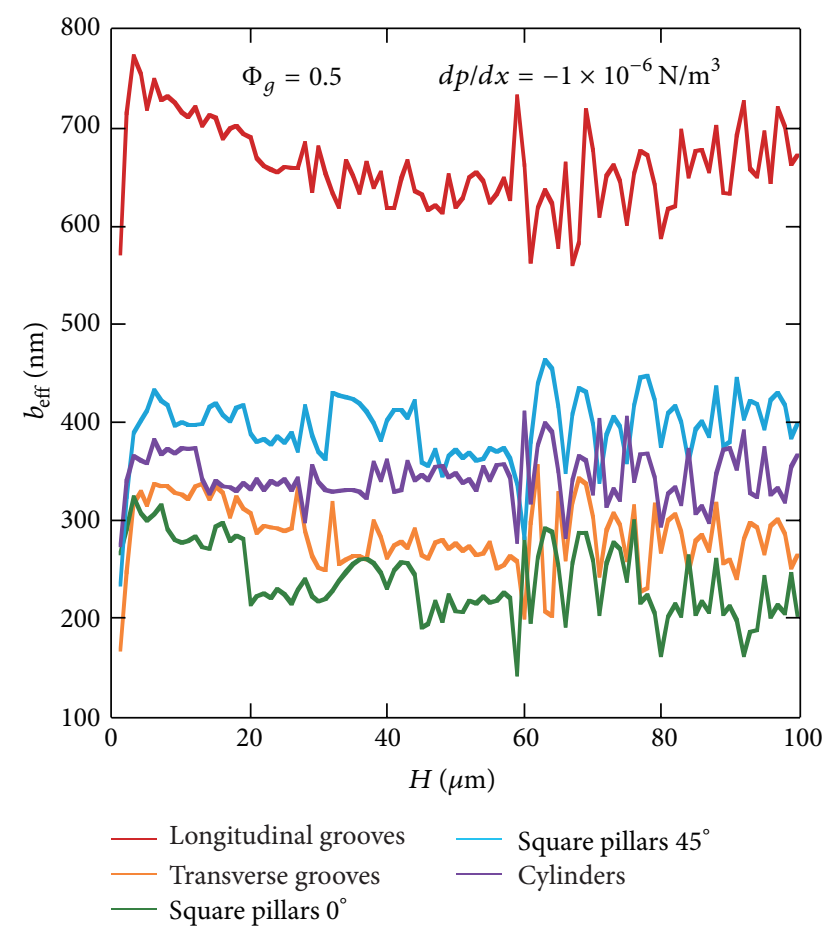

FIGURE 8: Relational graph of height of the channel to the effective slip.

that textures on surfaces can remarkably boost the flow in channels with height in small scales. Among the five cases, surface decorated with longitudinal grooves has the largest flow rate, which shows a better lubrication.

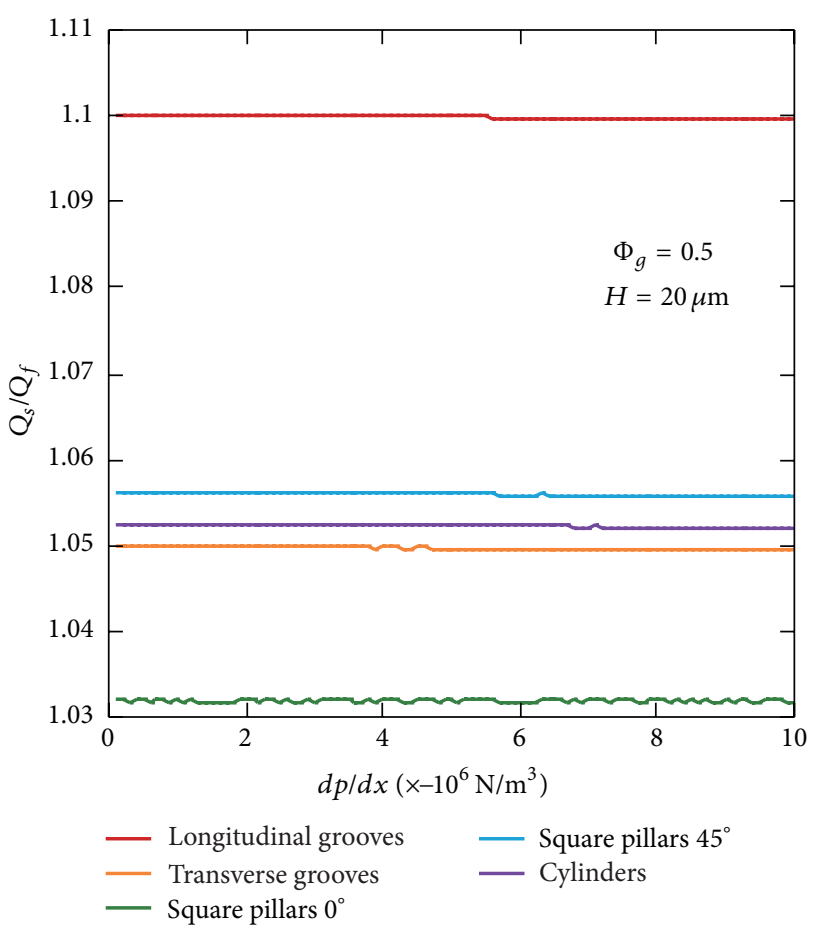

FIGURE 9: Relational graph of the pressure difference to the flow rate.

In Figure 8, the effective slip $b_{\text {eff }}$ shows a clear distinction: a sharp increase when $H$ increases from $1 \mu \mathrm{m}$ to about $5 \mu \mathrm{m}$; then the effective slip remains approximately constant as $H$ increase to $100 \mu \mathrm{m}$ but with visible fluctuations. It is noticeable that when $H$ is small, the upper wall with nonslip will have a strong effect on the flow near the lower superhydrophobic surfaces. With the increase of the height, this effect will decay until it is close to zero. When $H$ is larger than $20 \mu \mathrm{m}, b_{\text {eff }}$ fluctuates around a certain number (details shown in Table 2). The meshing in this FEM simulation should be the reason, with an increasing $H$; the simulation needs to reconstruct the mesh in every step, which leads to the deviations. Therefore, it can be believed that the height of the channel $H$ has no obvious effect on the effective slip at the interface of superhydrophobic surface and liquid. The results show that channel characterized with longitudinal grooves has the largest effective slip while channel with square pillars $0^{\circ}$ has the smallest effective slip. This can be explained by the obstruction effect of the textures as mentioned in effect of surface textures.

Fixing the fraction of gas-liquid area $\Phi_{g}$ at 0.5 and the height of the channel $H$ at $20 \mu \mathrm{m}$, the effect of driving pressure gradient $d p / d x$ on the flow rate and the effective slip were studied based on the models shown in Figure 2. $d p / d x$ ranges from $-1 \times 10^{5}$ to $-1 \times 10^{7} \mathrm{~N} / \mathrm{m}^{3}$ with the step length $-1 \times 10^{5} \mathrm{~N} / \mathrm{m}^{3}$. The results are shown in Figures 9 and 10 .

Figure 9 shows that the superhydrophobic surfaces significantly promote the flow, and the flow rate is larger in the channel characterized with longitudinal grooves which suggests lower drag and larger effective slip at the same driving pressure gradient, as shown in Figure 10. It is obvious that the effective slip on all kinds of surfaces descends slightly 


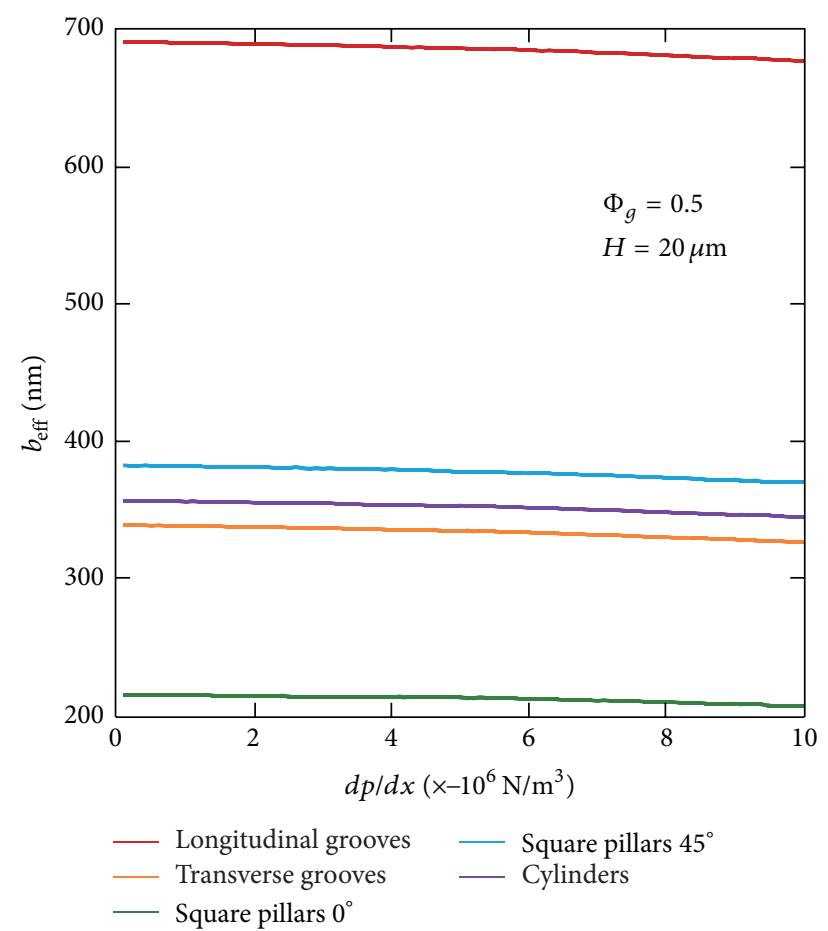

FIGURE 10: Relational graph of pressure difference to the effective slip.

with the increase of the driving pressure gradient. The data essentially reveal the degree of lubrication of the textures. Order of the intercepts of the five cases also can be explained by the obstruction effect of their shapes.

\section{Conclusion}

The flow rate in the channel with different textures, fraction of gas-liquid area, height of the channel, and driving pressure gradient are analyzed. Unlike most previous research, the independent effect of the four factors was studied, respectively. Textures with small angle of inclination to the direction of flow can reduce the drag and enhance the flow. The flow rate will increase more sharply with larger fraction of gasliquid which is in agreement with previous studies [19-24].

The results also show that the effective slip is affected by the surface textures and the gas-liquid fraction. The height of the channel and the change in the driving pressure gradient have little or no effect on the effective slip. The flow analysis showed that the flow on the surface with longitudinal grooves and higher fraction of gas-liquid exhibit less drag.

\section{Conflict of Interests}

The authors declare that there is no conflict of interests regarding the publication of this paper.

\section{Acknowledgment}

This research was supported by National Natural Science Foundation of China (Grants nos. 51475118 and 51505108).

\section{References}

[1] H. A. Stone, A. D. Stroock, and A. Ajdari, "Engineering flows in small devices: microfluidics toward a lab-on-a-chip," Annual Review of Fluid Mechanics, vol. 36, pp. 381-411, 2004.

[2] T. M. Squires and S. R. Quake, "Microfluidics: fluid physics at the nanoliter scale," Reviews of Modern Physics, vol. 77, no. 3, pp. 977-1026, 2005.

[3] D. Jing and B. Bhushan, "The coupling of surface charge and boundary slip at the solid-liquid interface and their combined effect on fluid drag: a review," Journal of Colloid and Interface Science, vol. 454, pp. 152-179, 2015.

[4] O. I. Vinogradova and A. V. Belyaev, "Wetting, roughness and flow boundary conditions," Journal of Physics: Condensed Matter, vol. 23, no. 18, Article ID 184104, 16 pages, 2011.

[5] G. McHale, M. I. Newton, and N. J. Shirtcliffe, "Immersed superhydrophobic surfaces: gas exchange, slip and drag reduction properties," Soft Matter, vol. 6, no. 4, pp. 714-719, 2010.

[6] O. I. Vinogradova and G. E. Yakubov, "Dynamic effects on force measurements. II. Lubrication and the atomic force microscope," Langmuir, vol. 19, no. 4, pp. 1227-1234, 2003.

[7] O. I. Vinogradova, "Slippage of water over hydrophobic surfaces," International Journal of Mineral Processing, vol. 56, no. 1, pp. 31-60, 1999.

[8] L. Bocquet and J.-L. Barrat, "Flow boundary conditions from nano- to micro-scales," Soft Matter, vol. 3, no. 6, pp. 685-693, 2007.

[9] E. Lauga, M. P. Brenner, and H. A. Stone, "Microfluidics: the noslip boundary condition," in Handbook of Experimental Fluid Dynamics, vol. 19, pp. 1219-1240, Springer, 2007.

[10] C. Sendner, D. Horinek, L. Bocquet, and R. R. Netz, "Interfacial water at hydrophobic and hydrophilic surfaces: slip, viscosity, and diffusion," Langmuir, vol. 25, no. 18, pp. 10768-10781, 2009.

[11] P. A. Thompson and S. M. Troian, "A general boundary condition for liquid flow at solid surfaces," Nature, vol. 389, no. 6649, pp. 360-362, 1997.

[12] C. Cottin-Bizonne, B. Cross, A. Steinberger, and E. Charlaix, "Boundary slip on smooth hydrophobic surfaces: intrinsic effects and possible artifacts," Physical Review Letters, vol. 94, no. 5, Article ID 056102, 4 pages, 2005.

[13] C. Ybert, C. Barentin, C. Cottin-Bizonne, P. Joseph, and L. Bocquet, "Achieving large slip with superhydrophobic surfaces, scaling laws for generic geometries," Physics of Fluids, vol. 19, no. 12, Article ID 123601, 10 pages, 2007.

[14] O. I. Vinogradova, K. Koynov, A. Best, and F. Feuillebois, "Direct measurements of hydrophobic slippage using doublefocus fluorescence cross-correlation," Physical Review Letters, vol. 102, no. 11, Article ID 118302, 5 pages, 2009.

[15] Y. Pan, B. Bhushan, and X. Zhao, "The study of surface wetting, nanobubbles and boundary slip with an applied voltage: a review," Beilstein Journal of Nanotechnology, vol. 5, no. 1, pp. 1042-1065, 2014.

[16] O. I. Vinogradova, N. F. Bunkin, N. V. Churaev, O. A. Kiseleva, A. V. Lobeyev, and B. W. Ninham, "Submicrocavity structure of water between hydrophobic and hydrophilic walls as revealed by optical cavitation," Journal of Colloid and Interface Science, vol. 173, no. 2, pp. 443-447, 1995.

[17] C. Cottin-Bizonne, J.-L. Barrat, L. Bocquet, and E. Charlaix, "Low-friction flows of liquid at nanopatterned interfaces," Nature Materials, vol. 2, no. 4, pp. 237-240, 2003. 
[18] S. Schmieschek, A. V. Belyaev, J. Harting, and O. I. Vinogradova, "Tensorial slip of superhydrophobic channels," Physical Review E, vol. 85, no. 1, Article ID 016324, 11 pages, 2012.

[19] A. Maali, Y. Pan, B. Bhushan, and E. Charlaix, "Hydrodynamic drag-force measurement and slip length on microstructured surfaces," Physical Review E, vol. 85, no. 6, Article ID 066310, 2012.

[20] C.-H. Choi, C.-J. Kim, and C. Lee, "Structured surfaces for a giant liquid slip," Physical Review Letters, vol. 101, no. 6, Article ID 064501, 2008.

[21] C.-H. Choi, U. Ulmanella, J. Kim, C.-M. Ho, and C.-J. Kim, "Effective slip and friction reduction in nanograted superhydrophobic microchannels," Physics of Fluids, vol. 18, Article ID 087105, 8 pages, 2006.

[22] P. Joseph, C. Cottin-Bizonne, J.-M. Benoît et al., "Slippage of water past superhydrophobic carbon nanotube forests in microchannels," Physical Review Letters, vol. 97, no. 15, Article ID 156104, 4 pages, 2006.

[23] J. Ou and J. P. Rothstein, "Direct velocity measurements of the flow past drag-reducing ultrahydrophobic surfaces," Physics of Fluids, vol. 17, no. 10, Article ID 103606, 2005.

[24] J. R. Philip, "Flows satisfying mixed no-slip and no-shear conditions," Zeitschrift für Angewandte Mathematik und Physik, vol. 23, no. 3, pp. 353-372, 1972.

[25] D. Quéré, "Wetting and roughness," Annual Review of Materials Research, vol. 38, pp. 71-99, 2008.

[26] J. Bico, C. Marzolin, and D. Quéré, “Pearl drops," Europhysics Letters, vol. 47, no. 2, pp. 220-226, 1999.

[27] A. L. Dubov, J. Teisseire, and E. Barthel, "Elastic instability and contact angles on hydrophobic surfaces with periodic textures," EPL, vol. 97, no. 2, Article ID 26003, 2012.

[28] C. Lee, C. H. Choi, and C. J. Kim, "Structured surfaces for a giant liquid slip," Physical Review Letters, vol. 101, no. 6, Article ID 064501, 4 pages, 2008.

[29] M. Z. Bazant and O. I. Vinogradova, "Tensorial hydrodynamic slip," Journal of Fluid Mechanics, vol. 613, pp. 125-134, 2008.

[30] F. Feuillebois, M. Z. Bazant, and O. I. Vinogradova, "Effective slip over superhydrophobic surfaces in thin channels," Physical Review Letters, vol. 102, Article ID 026001, 4 pages, 2009.

[31] J. P. Rothstein, "Slip on superhydrophobic surfaces," Annual Review of Fluid Mechanics, vol. 42, pp. 89-109, 2010.

[32] W. M. Haynes, Handbook of Chemistry and Physics, Academic Press, New York, NY, USA, 91st edition, 2010.

[33] O. I. Vinogradova, "Drainage of a thin liquid film confined between hydrophobic surfaces," Langmuir, vol. 11, no. 6, pp. 2213-2220, 1995.

[34] T. V. Nizkaya, E. S. Asmolov, and O. I. Vinogradova, "Flow in channels with superhydrophobic trapezoidal textures," Soft Matter, vol. 9, no. 48, pp. 11671-11679, 2013.

[35] D. Jing and B. Bhushan, "Electroviscous effect on fluid drag in a microchannel with large zeta potential," Beilstein Journal of Nanotechnology, vol. 6, no. 1, pp. 2207-2216, 2015.

[36] H. Ban, B. C. Lin, and Z. R. Song, "Effect of electrical double layer on electric conductivity and pressure drop in a pressuredriven microchannel flow," Biomicrofluidics, vol. 4, Article ID 014104, 14 pages, 2010.

[37] K. Ahmad, X. Zhao, Y. Pan, W. Wang, and Y. Huang, "Atomic force microscopy measurement of slip on smooth hydrophobic surfaces and possible artifacts," The Journal of Physical Chemistry C, vol. 119, no. 22, pp. 12531-12537, 2015.
[38] A. Gaddam, M. Garg, A. Agrawal, and S. S. Joshi, "Modeling of liquid-gas meniscus for textured surfaces: effects of curvature and local slip length," Journal of Micromechanics and Microengineering, vol. 25, no. 12, Article ID 125002, 13 pages, 2015.

[39] A. M. J. Davis and E. Lauga, "Geometric transition in friction for flow over a bubble mattress," Physics of Fluids, vol. 21, no. 1, Article ID 011701, 2009.

[40] A. Steinberger, C. Cottin-Bizonne, P. Kleimann, and E. Charlaix, "High friction on a bubble mattress," Nature Materials, vol. 6, no. 9, pp. 665-668, 2007. 

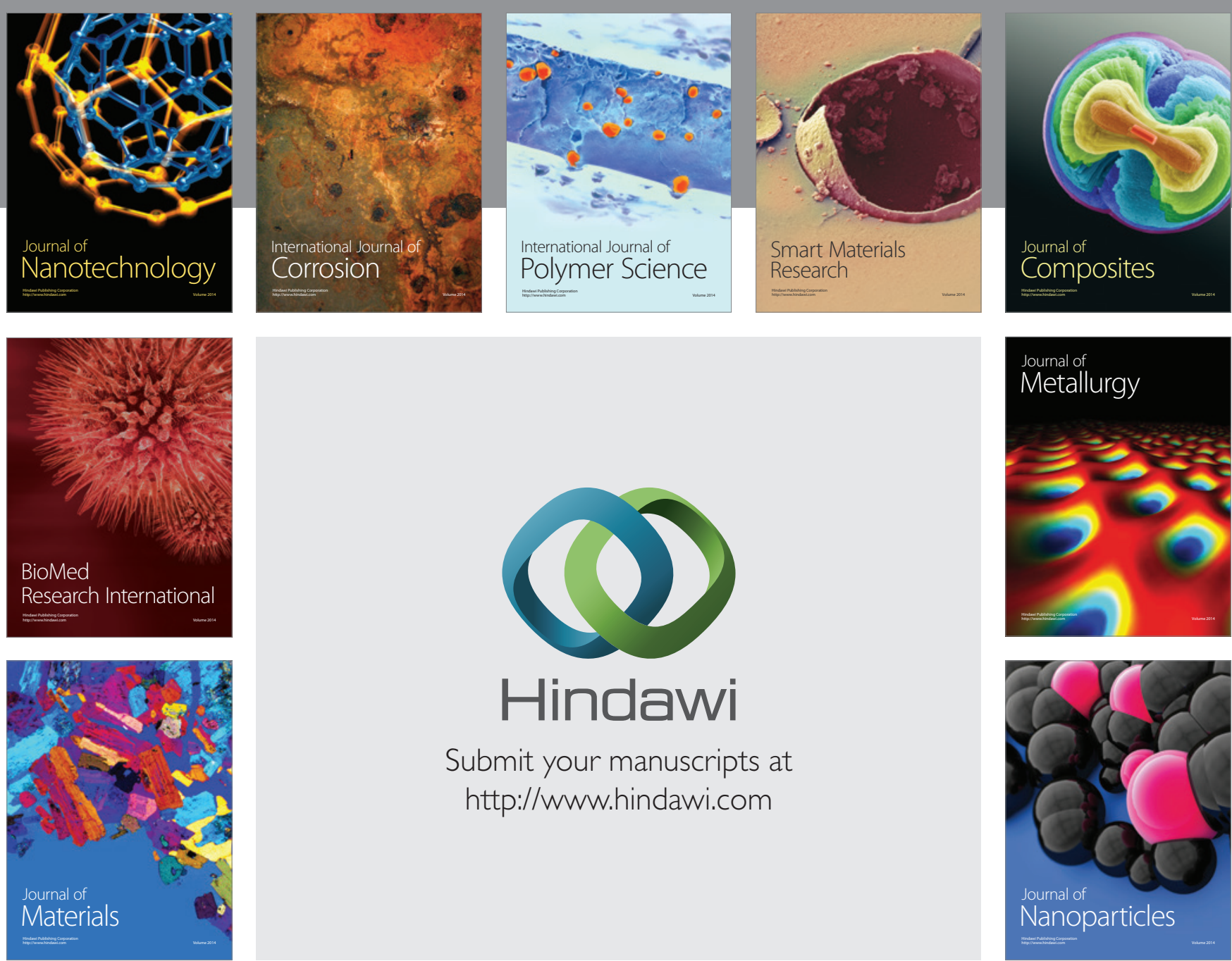

\section{Hindawi}

Submit your manuscripts at

http://www.hindawi.com

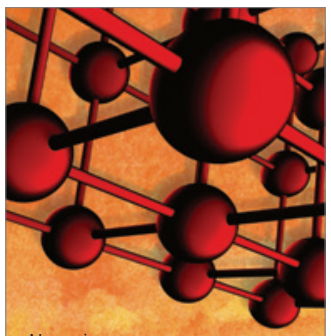

Materials Science and Engineering
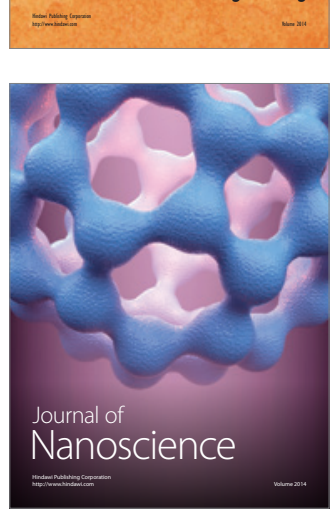
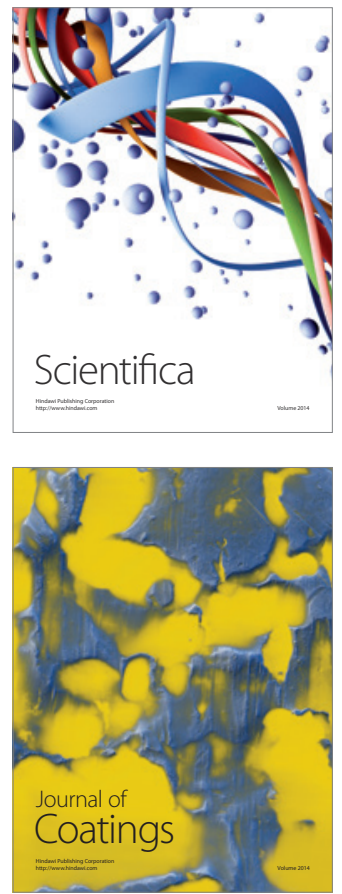
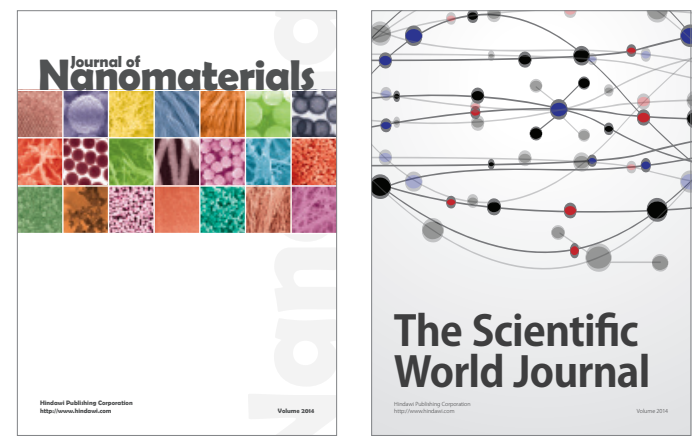

The Scientific World Journal
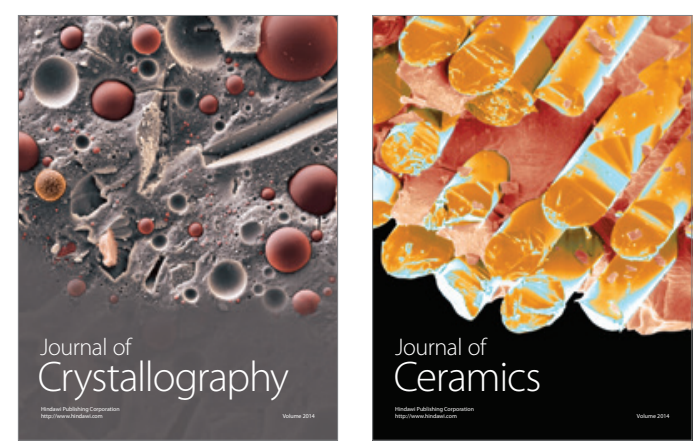
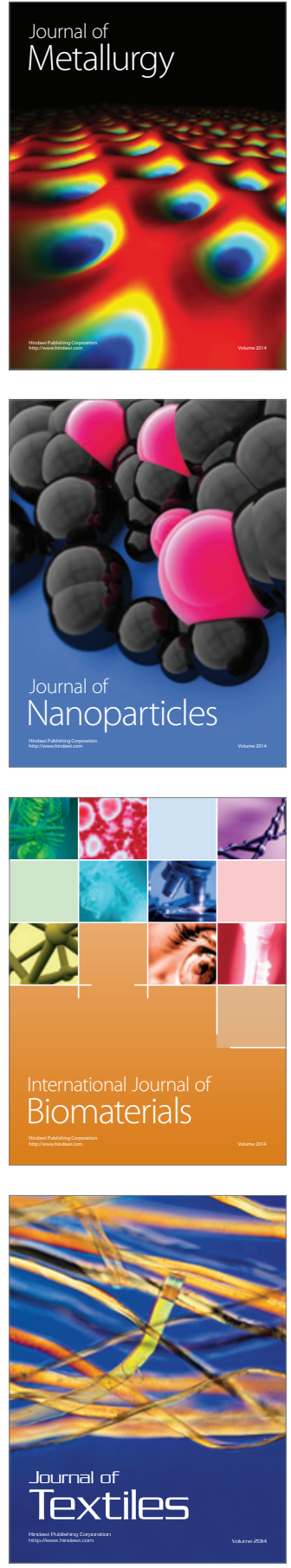